\title{
Basic study on a pseudo trend phenomenon of the Feynman- $\alpha$ analysis with bunching method
}

\author{
Sin-ya Hohara ${ }^{1 *}$, Atsushi Sakon ${ }^{1}$, Tomohiro Endo ${ }^{2}$, Tadafumi Sano ${ }^{1}$, \\ Kunihiro Nakajima ${ }^{3}$, Kazuki Takahashi ${ }^{3}$, Kengo Hashimoto ${ }^{1}$ \\ ${ }^{1}$ Atomic Energy Research Institute, Kindai University \\ 3-4-1 Kowakae, Higashi-Osaka city, Osaka, Japan \\ ${ }^{2}$ Nagoya University \\ Furo-cho, Chikusa-ku, Nagoya, Japan \\ ${ }^{3}$ Graduate School of Science and Engineering, Kindai University \\ 3-4-1 Kowakae, Higashi-Osaka city, Osaka, Japan
}

hohara@kindai.ac.jp, sakon@kindai.ac.jp,t-endo@energy.nagoya-u.ac.jp,t-sano@kindai.ac.jp, kunihiro_nakajima@nk-mail.com, takahashi.kazuki0524@gmail.com,kengoh@pp.iij4u.or.jp

\begin{abstract}
In these years, reactor noise analysis methods have been studied to apply for the Debris' criticality management at the Fukushima Daiichi NPP, Japan. The Feynman- $\alpha$ analysis with bunching method is one of the candidate techniques, however the bunching method itself has never been validated in detail. This synthesis technique is useful to reduce a time required for the experiment, however it is known that a non-physical trend unrelated to the state of a nuclear reactor is generated by the multiple use of time series data, and this phenomenon (we call "pseudo trend phenomenon") has never been systematically studied in detail. In this study, Poisson events, whose statistical characteristics were clarified, were employed to investigate the pseudo trend phenomenon of the bunching method. The time-sequence count data for various statistical parameters were generated by the Monte Carlo time series simulator. Comparing the two results obtained by applying the conventional bunching method and the moving-bunching method for the same Poisson event time series, and it was found that the same pseudo trend component appears in both results of the bunching method and the moving bunching method. In addition, it was also found that the fluctuation width of the pseudo trend component is smaller than the statistical fluctuation range.
\end{abstract}

KEYWORDS: Feynman- $\alpha$ analysis, bunching method, pseudo trend phenomenon

\section{INTRODUCTION}

In recent years, in Japan, the development of criticality safety assessment methods for fuel debris at the TEPCO Fukushima Daiichi Nuclear Power Plant (hereinafter referred to as "1F") has become a topic. The Nuclear Power Plant Accident occurred on the 1F due to an extreme big earthquake in March 2011, causing core melting. The melted core is present as fuel debris in the containment bottom and continues to cool by the insertion and circulation water, but it has not yet reached its extraction. Since the debris containing uranium is present in the cooling water, criticality safety assessment for the debris is a technique required 
during removal work. On the other hand, in the site of $1 \mathrm{~F}$, the effects of the accident in 2011, unlike the normal abolition process, voids for loading the instrument for criticality safety assessment can't be sufficiently secured. For this reason, the topic is to develop a criticality safety assessment method that can be performed with fewer cable loads.

In the nuclear community in Japan, the reactor noise technique is listed as one of the candidate technologies for $1 \mathrm{~F}$ debris criticality safety assessment ${ }^{[1]}$ and in the United States, reactor noise analysis technology has been promoted as a sub criticality measurement technology for the underwater core. ${ }^{[2]}$ Feynman- $\alpha$ method is one of the leading candidates for criticality management method of $1 \mathrm{~F}$ debris. When applying the Feynman- $\alpha$ method, bunching method for the purpose of shortening the measurement time is used in combination as a de facto standard. When the bunching method is applied for the data analysis, the fluctuation of the Feynman- $\alpha$ plot becomes smaller than the theoretical statistics error range. ${ }^{[3][4]}$ In addition, there are some unclear strange effects on the bunching method.

As those observed as the most typical influence by the bunching method, there is a trend component that occurs with respect to the time gate direction as described above, it has been confirmed that does not occur when not applying the bunching method. ${ }^{[4][5]}$ This trend component (we call it a "pseudo trend") is not present in Feynman's theory and has a dependency component of more than the fluctuation width for time gate, but shows a different tendency in each observation time series even under the same core conditions. Pseudo trend component, since it tends to decrease when the measurement time is longer, it is less likely to be a problem in the normal observation of the core. However, observation of fuel debris, etc., in the observation at a very deep sub-critical situation, pseudo trend component may be a size that can't be ignored.

In this study, in order to clarify the characteristics of pseudo trend phenomena that occur when the bunching method is applied, we investigated using Poisson event which has clear characteristics. In this paper, we describe the characteristics of pseudo trend phenomena that are clearly present in the research. Section 2 outlines the Feynman- $\alpha$ and Bunching methods, and Section 3 describes the methods and calculation conditions of the investigation. Section 4 shows the results obtained and concludes in Section 5.

\section{THEORY}

The Pseudo trend phenomenon is expressed when using repeatedly the same time series data on Feynman$\alpha$ method. In this section an overview of the Feynman- $\alpha$ method, bunching method and Pseudo trend phenomenon are provided.

\subsection{Feynman- $\alpha$ method and Bunching method}

Feynman- $\alpha$ method is a method for observing sub-criticality in neutron multiplying systems and was devised by R. P. Feynman. ${ }^{[6]}$ In this method, the deviation from Poisson statistics of the counting distribution of neutrons in the multiplying system is observed through the Variance-to-Mean ratio, and the prompt neutron decay constant $\alpha$ is observed from the dependency between the counting time (so called "Time gate") and the ratio. Theoretically, the Variance-to-Mean ratio VtoM(t) is observed in accordance with the following formula as the synthesis of the deviation components of each group neutrons (prompt neutron and delayed neutron) and Poisson events' component (=1).

$$
\operatorname{VtoM}(t)=1+\sum_{i=0}\left\{Y_{i} \cdot\left(1-\frac{1-\exp \left(-\omega_{i} \cdot t\right)}{\omega_{i} \cdot t}\right)\right\}
$$

where $t$ is time gate, $\omega_{i}$ is a decay constant of $i$-th group neutron and $Y_{i}$ is a saturation factor of $i$-th group neutron. Compared to the time scale of the delayed neutron component, the time scale of the prompt neutron component is significantly biased to the high frequency region. In practice, one is interested in the prompt neutrons and their decay constant that is related to the reactivity of the system through the relation $\alpha=$ 
$(\beta-\rho) / \Lambda$, where $\beta$ is the total delayed neutron fraction, $\rho$ is the reactivity and $\Lambda$ is the generation time. Therefore, one generally restricts the Feynman- $\alpha$ analysis to small time gates, where the delayed neutron component is often approximated as a linear function of the time gate $t$ as in [7]:

$$
\operatorname{VtoM}(t)=1+\left\{Y_{\infty} \cdot\left(1-\frac{1-\exp (-\alpha \cdot t)}{\alpha \cdot t}\right)+C_{1}+C_{2} \cdot t\right\}
$$

where $\alpha$ is a prompt neutron decay constant, $Y_{\infty}$ is a saturation factor of prompt neutron and $C_{1}, C_{2}$ are approximate factors for the delayed neutron. Ideally it is necessary to perform the measurement independently at each time gate, a very long measurement time is needed in order to extract $\alpha$ from the Variance-to-Mean ratio. Around 1990, a bunching method was devised to solve this problem. ${ }^{[8]}$ The bunching method is a data processing method using the same time series repeatedly for all time gate as shown in Figure 1. The bunching method succeeded in significantly shortening the time of Feynman- $\alpha$ method experiments about one hundredth. Furthermore, as shown in Figure 2, a method called moving bunching method for "earning" more samples from the same time series data has also been devised. ${ }^{[9]}$

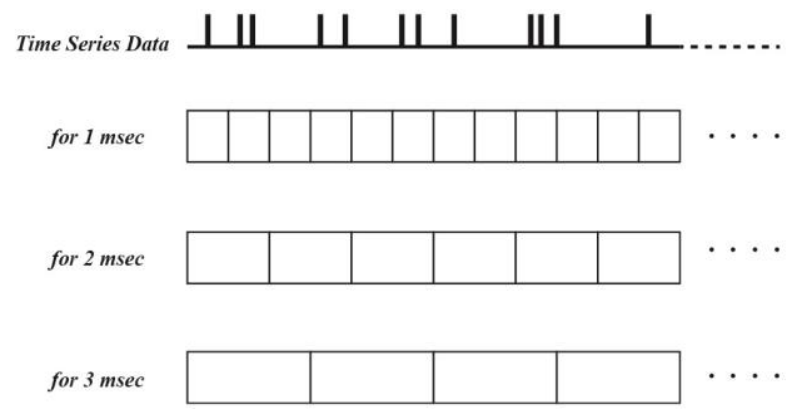

Figure 1. Schematic view of the Bunching method

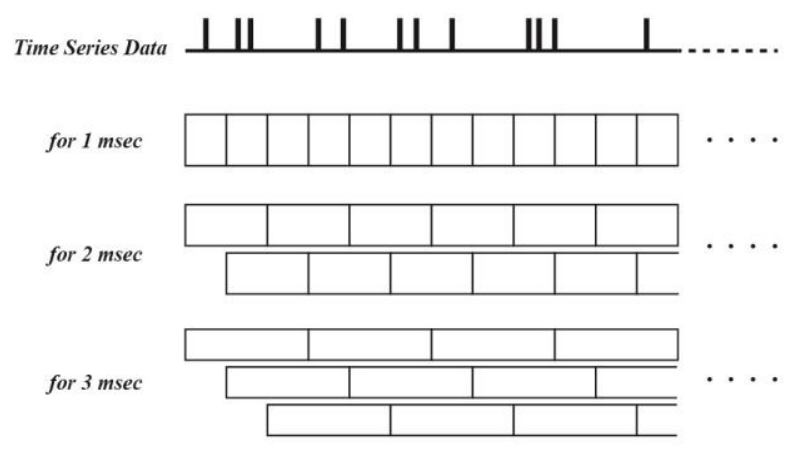

Figure 2. Schematic view of the Moving Bunching method

\subsection{Pseudo trend Phenomenon}

As described above, the data processing method of the bunching method and its derivation, the experimental time required for Feynman- $\alpha$ experiment was greatly shortened. However, the theoretical background of the same result as the original Feynman- $\alpha$ method did not exist for the data processing methods. Feynman's theory did not include repeated use of a time series data. Repeatedly using the same time series data for 
evaluating the variance-to-mean ratio for several time gate values create correlations between these values, whose magnitude has been observed to be smaller than the inherent statistical fluctuation of the process. In addition, as described above, as another aspect of repeated use of time series data, it is also known to form a pseudo trend. When performing observation for the actual reactor, the result is obtained as a mixture of the component of equation (2) and pseudo trend component which is different for each time series data as found in Figure 3.

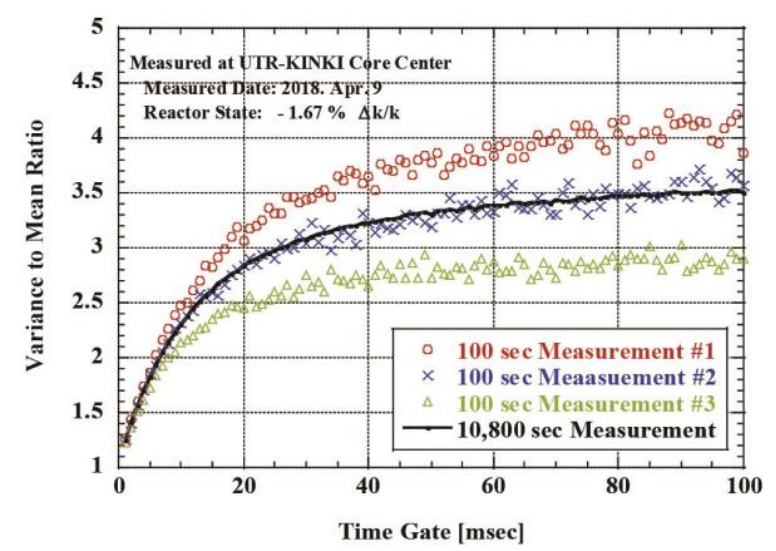

Figure 3. An example of measurement result for actual reactor

However, there is only a little research about this pseudo trend phenomenon itself. Therefore, as described in the next section, we investigated the characteristics of Pseudo trend phenomena using the Poisson event that has a clear characteristic.

\section{INVESTIGATION METHOD}

\subsection{Simulation of Poisson Events by Computer}

Because the measured signals of a multiplying media - especially with high sub-criticality - only slightly differ from a Poisson distribution, and because the characteristics of the Poisson distributions are wellknow, we use pure Poisson signals to investigate Pseudo trend phenomena using Poisson events generated by computers.

In a Poisson process, the interarrival times, i.e. the times between two consecutive arrivals or events, are independent and follow an exponential function. ${ }^{[10]}$ Therefore, it is possible to simulate the time interval of Poisson event of the counting rate $r$ from a uniform random number using the inverse integral function ${ }^{[11]}$ as the following equation.

$$
T_{\text {interval }}=-\frac{1}{r} \times \log (\operatorname{Rand}[0 \sim 1])
$$

where $T_{\text {interval }}$ is a time interval and Rand [0 1] is a uniformed random number between 0 and 1 . The time interval is integrated and the time series data for the investigation is generated. The virtual numerical experiment was performed using originally constructed code developed on Micro Soft Visual Studio 2015/C\#, and using a random number generated by the Random class based on a modified version of Donald E. Knuth's subtractive random number generator algorithm. 


\section{2. $\mathrm{Z}$ transformation of Mean, Variance, Variance-to-Mean Ratio}

As with actual experiments and analyses, the same generated time series data is used for all time gates in the investigation. Therefore, the number of samples is different in the short time gate and the long-time gate. Therefore, the number of samples for each time gate is different in the normal Feynman- $\alpha /$ bunching analysis. This causes the different statistic fluctuation range for each time gate, and it might be a problem with the investigation of the Pseudo trend phenomenon. To solve this problem, it is necessary to standardize in the standard deviation (so called z-transformation) at each time gate. When the events follow a Poisson process, the standard deviations of the empirical mean, empirical variance, and empirical Variance-toMean ratio are written with the mean count $\bar{N}=\sum_{i} N_{i} / n$ as follows. ${ }^{[12]}$

$$
\begin{aligned}
& \delta_{\text {Mean }}=\sqrt{\bar{N} / n} \\
& \delta_{\text {Variance }}=\sqrt{\left(\frac{1}{n}+\frac{2}{n-1} \cdot \bar{N}\right) \cdot \bar{N}} \\
& \delta_{\text {VtoM }}=\sqrt{\frac{2}{n-1}}
\end{aligned}
$$

where $\delta_{\text {Mean }}, \delta_{\text {Variance }}, \delta_{V t o M}$ are standard deviations of mean, variance, Variance to Mean Ratio, $n$ is a number of the sample gate for a given time gate width..

Each obtained value is z-transformed with following formula.

$$
z \text { value }=\frac{\text { Calculated Value-Theoretical Value }}{\text { Standard Deviation }}
$$

\subsection{Calculation Parameters}

We investigate pseudo trend phenomena using the Poisson events generated above. The parameters of the Poisson event generated by the computer are shown in the following table.

Table I. Parameters of the Poisson Event's generation

\begin{tabular}{|c|c|}
\hline Item & Value \\
\hline Event Rate $[\mathrm{cps}]$ & 100 \\
\hline Time Length $[\mathrm{sec}]$ & 1,000 \\
\hline Time gate $[\mathrm{msec}]$ & $1-1000$ (every $1 \mathrm{msec})$ \\
\hline
\end{tabular}

\section{RESULTS}

\subsection{Dependency on Mean, Variance and Variance-to-Mean Ratio after the $Z$ transformation}

A correlation diagram example between z-value of Mean, Variance and z-value of Variance-to-Mean ratio for the same time series data is shown in Figure 4. The analysis result is of the normal bunching method, and the analysis results for $1 \mathrm{msec}$-time gate to $1,000 \mathrm{msec}$-time gate are plotted in Figure 4 . There is no 
correlation between the Mean and the Variance-to-Mean ratio, however the variance and Variance-to-Mean ratio have a very strong correlation as found in Figure 4. From this, the pseudo trend component is mainly considered to propagate from the variance.

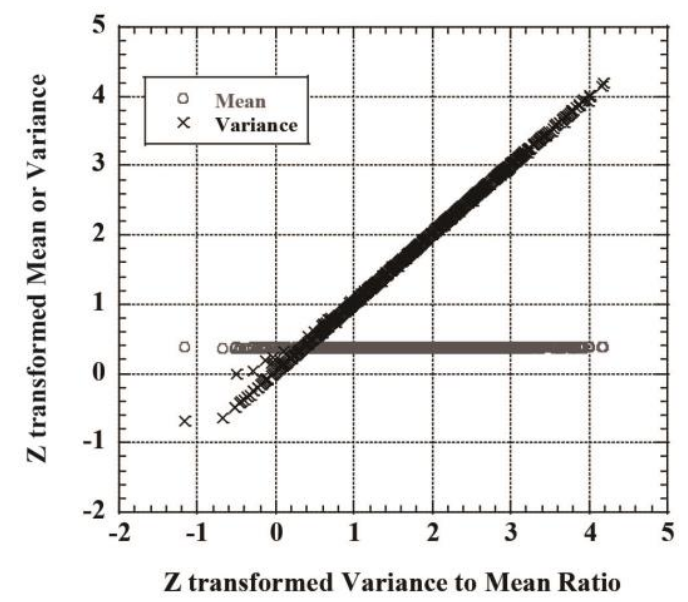

Figure 4. An example of correlation between Mean, Variance and Variance-to-Mean ratio

\subsection{Relationship between Bunching method and Moving Bunching method}

A comparative example of the analysis results of the bunching method and the moving bunching method after the $\mathrm{Z}$ transformation is shown in Figure 5. The difference between the result of the bunching method and the moving bunching method after the $\mathrm{Z}$ transformation is shown in Figure 6. It can be found that the result of the moving bunching method is located at the center of the result of the bunching method in Figure 5. Further, in Figure 6, it can be found that the difference between the results of the bunching method and the moving bunching method is uniformly existing in the range of -1 to 1 . From these, pseudo trend component for the same time series data appearing in both the bunching method and the moving bunching method is the same, and it was found that it is possible to extract the component containing the pseudo trend component by the moving bunching method.

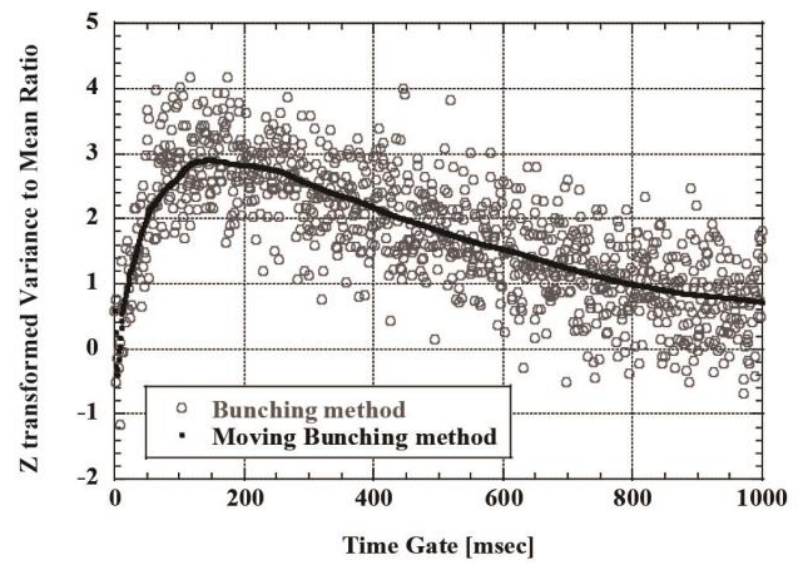

Figure 5. An example of comparison of the $Z$ transformed results of bunching method and moving bunching method 

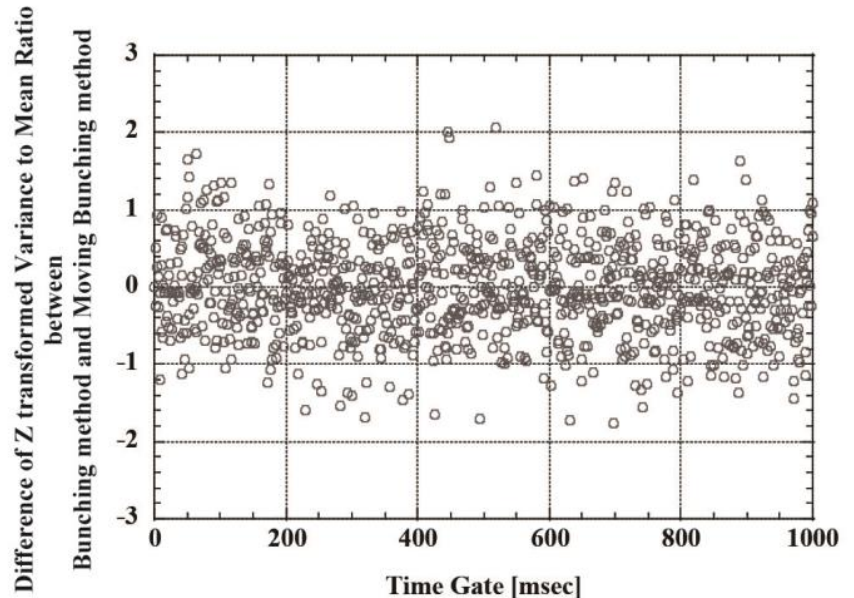

Figure 6. An example of difference between the $Z$ transformed results of bunching method and moving bunching method

Figure 7 shows a histogram of the Variance-to-Mean ratio after the $\mathrm{z}$ transformation. Figure 7-(a) shows the $\mathrm{Z}$ transformed results of the bunching method, and Figure 7-(b) shows the $\mathrm{Z}$ transformed results of the moving bunching method. The histogram is plotted using 10k time series data for each analysis method. The plotted histograms were fit with the gaussian, and the deviations of each histogram are shown in the chart legend. As shown in Figure 7, the processing result of the bunching method is comparable to the standard deviation, however the processing result of the moving bunching method fits in a range smaller than the statistical fluctuation range. Since the Poisson event is used in this study, the fluctuation of the trend component projected in Figure 7-(b) is of the pseudo trend component itself. In addition, the fluctuation of the pseudo trend was found to stay in a smaller area than the statistical fluctuation range as shown in Figure 7-(b).
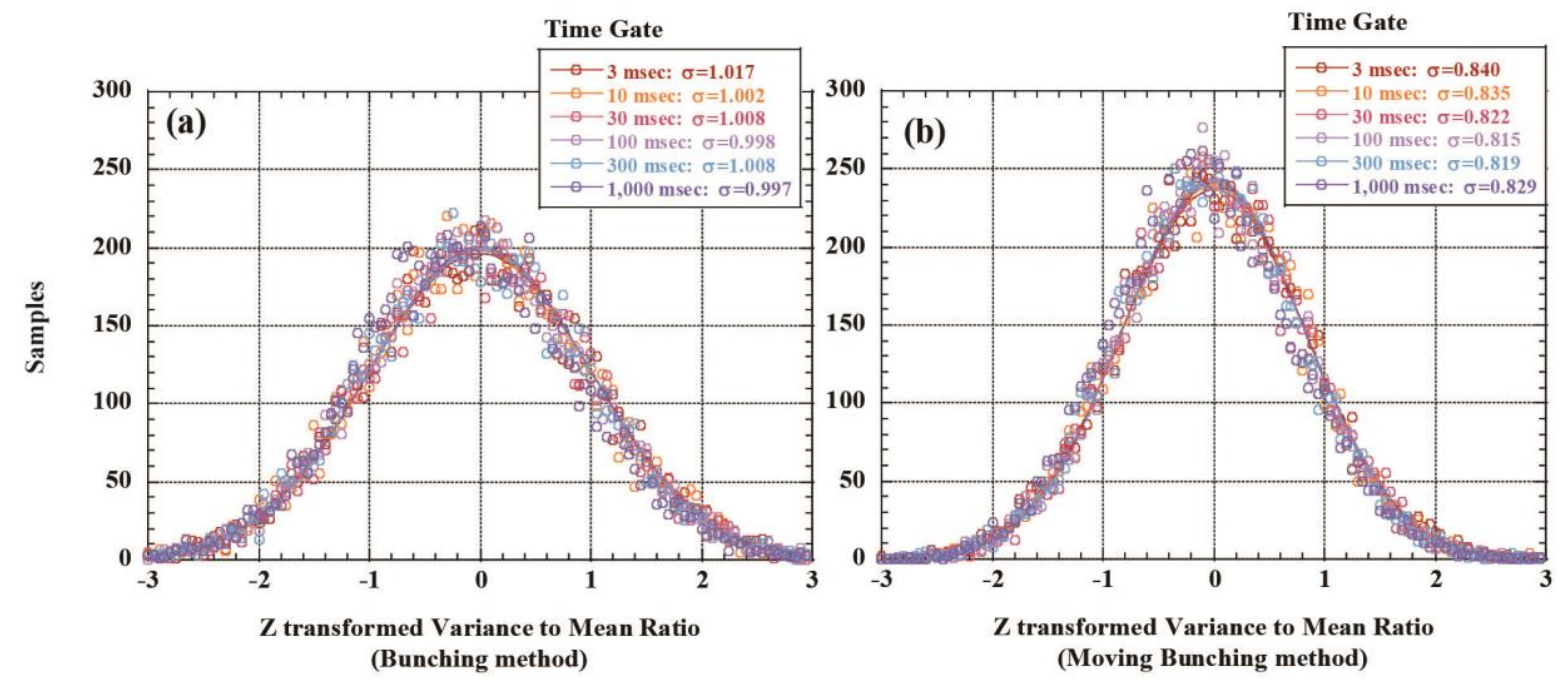

Figure 7. Histograms of the $\mathrm{Z}$ transformed Variance-to-Mean ratio 


\section{CONCLUSION}

In this work, we investigated the basic characteristics of pseudo trend phenomena occurring in Feynman$\alpha /$ bunching method, one of the reactor noise analysis method. We used Poisson events generated by the computers to investigate the analysis results of the bunching method and the moving bunching method. As the result, it was found that pseudo trend component appearing in the Variance-to-Mean ratio is mainly propagated from variance. In addition, it was found that the same pseudo trend component appears in both results of the bunching method and the moving bunching method. Finally, it was also found that the fluctuation width of the pseudo trend component is smaller than the statistical fluctuation range. However, the fundamental structure of the pseudo trend phenomenon has not yet been clarified. Further investigation is needed to understand the pseudo trend phenomena itself.

\section{REFERENCES}

1. S. Wada, S. Kano, T. Misawa, Y. Kitamura, "Sub-Criticarity Monitoring System for The Retrieval of Fuel Debris in Fukushima Dai-ichi Nuclear Power Plants", Proceedings of ICNC $2019-11^{\text {th }}$ International conference on Nuclear Criticality Safety, Paris, France, 15-20 September 2019

2. J. Arthur, R. Bahran, J. Hutchinson, A. Sood, N. Thompson, S. A. Pozzi, "Development of a research reactor protocol for neutron multiplication measurements", Progress in Nuclear Energy, Vol. 106, pp120-139 (2018)

3. P. Humbert, "Simulation and Analysis of List Mode Measurements on SILENE Reactor", Journal of Computational and Theoretical Transport, Vol. 47, pp350-363 (2018)

4. T. Endo, A. Yamamoto, "Comparison of theoretical formulae and bootstrap method for statistical error estimation of Feynman- $\alpha$ method", Annals of Nuclear Energy, Vol. 124, pp606-615 (2019)

5. S. Hohara, K. Nakajima, A. Sakon \& K. Hashimoto, "An applied limit of the bunching method for the Feynman- $\alpha$ analysis", Journal of Nuclear Science and Technology, vol.55, pp1309-1316 (2018)

6. R. P. Feynman, F. de Hofman, R. Seber, "Dispersion of the Neutron Emission in U-235 Fission", $J$. Nucl. Energy, 3, pp6 (1956)

7. K. HASHIMOTO, T. MOURI \& N. OHTANI, "Reduction of Delayed-Neutron Contribution to Variance-to-Mean Ratio by Application of Difference Filter Technique", Journal of Nuclear Science and Technology, vol.36, pp555-559 (1998)

8. T. Misawa, S. Shiroya, K. Kanda., "Measurement of Prompt Decay Constant and Subcriticality by the Feynman- $\alpha$ Method", Nuclear Science and Engineering, vol. 104, pp53-65 (1990)

9. R. Okuda, A. Sakon, S. Hohara, W. Sugiyama, H. Taninaka \& K. Hashimoto, "An improved Feynman- $\alpha$ analysis with a moving-bunching technique", Journal of Nuclear Science and Technology, vol.53, pp1647-1652 (2016)

10. G. F. Knoll, Radiation Detection and Measurement Third Edition, pp97, John Wiley \& Sons Inc, New York, United States (2000)

11. W. H. Press, S. A. Teukolsky, W. T. Vetterling, B. P. Flannery, Numerical Recipes in C Second Edition, pp287, Cambridge University Press, New York, United States (1992)

12. Y. Dodge \& V. Rousson, "The Complications of the Fourth Central Moment", The American Statistician, Vol. 53, pp. 267-269 (1999) 